\title{
SKIN ANTI-AGING STRATEGIES: A
}

\section{REVIEW}

\author{
Sheetal Mane, Kuldeep Vinchurkar, Maheer Khan, Jitendra Sainy, Surekha Nirmal, Renu Singh \\ Devi Ahilya Vishwavidyala, Indore (M.P) \\ Indore Institute of Pharmacy, Indore (M.P.)
}

\begin{abstract}
Skin aging is a complex biological process influenced by a combination of endogenous (intrinsic) and exogenous (extrinsic) factors. Because of the fact that skin health and beauty is considered one of the principal factors representing overall "well-being" and the perception of "health" in humans, several antiaging strategies have been developed during the last years. It is the intention of this article to review the most important anti-aging strategies that dermatologists have nowadays in hand, including preventive measurements, cosmetological strategies, topical and systemic therapeutic agents and invasive procedures.
\end{abstract}

Keywords: Aging, anti-aging, antioxidants, laser, peeling, fillers, botulinum toxin.

\section{INTRODUCTION}

Skin aging is a part of a natural human "aging mosaic" which becomes evident and follows different trajectories in different organs, tissues and cells with time. While the aging signs of internal organs are masked from the ambient "eyes," the skin provides first obvious marks of the passing time.

Skin aging is a complex biological process influenced by combination of endogenous (genetics, cellular metabolism, hormone and metabolic processes) and exogenous (chronic light exposure, pollution, ionizing radiation, chemicals, toxins) factor [1]. These factors lead together to cumulative structural and physiological alterations and progressive changes in each skin layer as well as changes in skin appearance, especially, on the sun-exposed skin areas [2]. In contrast to thin and atrophic, finely wrinkled and dry intrinsically aged skin, premature photoaged skin typically shows a thickened epidermis, mottled discoloration, deep wrinkles, laxity, dullness and roughness. Gradual loss of skin elasticity leads to the phenomenon of sagging Slowing of the epidermal turnover rate and cell cycle lengthening coincides with a slower wound healing and less effective desquamation in older adults. This fact is important when esthetic procedures are scheduled [3]. On the other side, many of these features are targets to product application or procedures to accelerate the cell cycle, in the belief that a faster turnover rate will yield improvement in skin appearance and will speed wound healing. A marked loss of fibrillinpositive structures as well as a reduced content of collagen type VII (Col-7), may contribute to wrinkles by weakening the bond between dermis and epidermis of extrinsically age skin [4]. Sunexposed aged skin is characterized by the solar elastosis. The sparse distribution and decrease in collagen content in photo aged skin can be due to increased collagen degradation by various matrix metalloproteinase's, serine, and other proteases irrespective of the same collagen production [5]. In older skin, collagen looks irregular and disorganized; the ratio of Col-3, to Col- 1 has been shown to increase, due, significantly, to a loss of Col-1. The overall collagen content per unit area of the skin surface is known to decline approximately $1 \% / y e a r$ [6]. Glycosaminoglycans (GAGs) are among the primary dermal skin matrix constituents assisting in binding water. In photo-aged skin, GAGs may be associated with abnormal elastotic material and thus be unable to function effectively. The total hyaluronic acid (HA) level in the dermis of skin that age intrinsically remains stable; however, epidermal HA diminishes markedly [6]. Three primary structural components of the dermis, collagen, elastin and GAGs have been the subjects of the majority of anti-aging research and efforts for aesthetic-anti-aging strategies pertaining to the skin, from "anti-wrinkle creams" to various filling agents [7].

Presentation of aging of the entire face is associated with the gravity impact, muscles action, loss of volume, diminishing and redistribution of superficial and deep fat, loss of bony skeleton support what all together lead to the face sagging, changes in shape and contour. Regardless of the fact that aging is a biological inevitable process and not a pathological condition it is correlated with various skin and body pathologies, including degenerative disorders, benign and malignant neoplasms.

The 'successful aging' paradigm, focuses on health and active participation in life, counters traditional 
conceptualizations of aging as a time of disease and is increasingly equated with minimizing age signs on the skin, face and body [8]. From this perspective, preventative aesthetic dermatology might supplement the request for healthy aging, treat or prevent certain cutaneous disorders, notably skin cancer, and delay skin aging combining local and systemic methods of therapy, instrumental devices and invasive procedures [9]. The mainspring of any skin anti-aging therapy is to achieve a healthy, smooth, blemish-free, translucent and resilient skin. In clinical practice, "to look better" doesn't mean to "look younger." That is why it is so important to understand patients' wishes and to orientate them to the treatment modality that will give the most satisfying results whereas knowing all available treatment techniques [10]. The age, previous procedures or surgery, general health status, type of the skin, style of life and many other factors should be taken into consideration before choosing the strategy for the individual case [11]. The desired therapeutic anti-aging effect of the skin is continuous, step-by step process, which combines various methods of the skin bio-revitalization and rejuvenation, augmentation, restoration of each skin layer individually and in the light of many other factors - from a style of the life to the immune, genetic, emotional and health status in general [12].

\section{SKIN AGING PREVENTION AND THERAPY}

The skin anti-aging strategies attempted to reverse the dermal and epidermal signs of photo- and chronological aging can be grouped under the following approaches (Table 1).

Table - 1 Skin antiaging approaches

\begin{tabular}{|l|l|}
\hline Cosmetological care & $\begin{array}{l}\text { Daily skin care } \\
\text { Correct sun protection } \\
\text { Aesthetic non-invasive } \\
\text { procedures }\end{array}$ \\
\hline $\begin{array}{l}\text { Topical medicine } \\
\text { agents or topical } \\
\text { agents }\end{array}$ & $\begin{array}{l}\text { Antioxidants } \\
\text { Cell regulators }\end{array}$ \\
\hline
\end{tabular}

\begin{tabular}{|l|l|}
\hline Invasive procedures & $\begin{array}{l}\text { Chemical peelings } \\
\text { Visible light devices } \\
\text { Intense pulsed light (IPL) } \\
\text { Ablative and nonablative } \\
\text { laser photo-rejuvenation } \\
\text { Radiofrequency (RF) } \\
\text { Injectable skin } \\
\text { biostimulation and } \\
\text { rejuvenation } \\
\text { Prevention of dynamic } \\
\text { wrinkles } \\
\text { Correction of static, } \\
\text { anatomical wrinkles } \\
\text { Restoration } \\
\text { (redistribution) of fat and } \\
\text { volume loss, skin } \\
\text { augmentation and } \\
\text { contouring }\end{array}$ \\
\hline $\begin{array}{l}\text { Systemic agents } \\
\text { avoiding } \\
\text { exogenous factors of } \\
\text { life style and habits } \\
\text { of }\end{array}$ & $\begin{array}{l}\text { Hormone replacement } \\
\text { therapy } \\
\text { Antioxidants }\end{array}$ \\
\hline $\begin{array}{l}\text { Smoking } \\
\text { Pollution } \\
\text { Stress } \\
\text { Nutrition, diet restriction } \\
\text { and alimentary } \\
\text { supplementation } \\
\text { Physical activity } \\
\text { Control of general health }\end{array}$ \\
\hline
\end{tabular}

Skin Care: Healthy and functioning skin barrier is important protector against dehydration, penetration of various microorganisms, allergens, irritants, reactive oxygen species and radiation. The skin barrier may be specifically adjusted to allow penetration. For this reason daily skin care may increase skin regeneration, elasticity, smoothness, and thus temporarily change the skin condition [13]. However, it is necessary to stop the degradation of the skin primary structural constituents, such as collagen, elastin, to prevent the formation of wrinkles. Although the technology required to suitably deliver these compounds into the skin has not yet been developed, some products do promote the natural synthesis of these substances except elastin enhancing. Another integral approach preventing wrinkle formation is the reduction of inflammation by topical or systemic antioxidants which should be used in combination with sunscreens and retinoids to enhance their protective effects.

Photoprotection and Systemic Antioxidants: Chronic photodamage of the skin manifests itself as extrinsic skin aging (photoageing). DNA 


\section{International Journal of Engineering Applied Sciences and Technology, 2019 Vol. 4, Issue 7, ISSN No. 2455-2143, Pages 255-263 \\ Published Online November 2019 in IJEAST (http://www.ijeast.com)}

photodamage and UV-generated reactive oxygen species (ROS) are the initial molecular events that lead to most of the typical histological and clinical manifestations of chronic photodamage of the skin. Wrinkling and pigmentary changes are directly associated with premature photo-aging and are considered its most important cutaneous manifestations. The strategies aimed at preventing photo-aging include sun avoidance, sun protection using sunscreens to block or reduce skin exposure to UV radiation, retinoids in order to inhibit collagenase synthesis and to promote collagen production, and anti-oxidants, particularly in combination, to reduce and neutralize free radicals (FR) [14].

Interventional studies indicate that it is in fact possible to delay skin aging and to improve skin conditions through administration of selected nutritional supplements. Nutritional antioxidants act through different mechanisms and in different compartments, but are mainly FR scavengers: (1) they directly neutralize FRs, (2) they reduce the peroxide concentrations and repair oxidized membranes, (3) they quench iron to decrease ROS production, (4) via lipid metabolism, short-chain free fatty acids and cholesteryl esters neutralize ROS. Endogenous antioxidant defenses are both non-enzymatic (e.g., uric acid, glutathione, bilirubin, thiols, albumin, and nutritional factors, including vitamins and phenols) and enzymatic [e.g., superoxide dismutases, glutathione peroxidases (GSHPx), and catalase]. The most important source of antioxidants is provided by nutrition. To the most known systemic antioxidants belong vitamin $\mathrm{C}$, vitamin $\mathrm{E}$, carotenoids, and from the trace elements copper and selenium [15]. There are also studies demonstrating that vitamins $\mathrm{C}$ and E combined with ferulic acid impart both a sunscreen and an anti-oxidant effect.

Topical Pharmacological Agents with AntiAging Properties: There are two main groups of agents that can be used as anti-aging cream components, the antioxidants and the cell regulators. The antioxidants, such as vitamins, polyphenols and flavonoids, reduce collagen degradation by reducing the concentration of FR in the tissues. The cell regulators, such as retinols, peptides and growth factors (GF), have direct effects on collagen metabolism and influence collagen production.

Vitamins $\mathrm{C}, \mathrm{B}_{3}$, and $\mathrm{E}$ are the most important antioxidants because of their ability to penetrate the skin through their small molecular weight [16]. The water-soluble, heat-labile local L-ascorbic acid (vitamin $\mathrm{C}$ ) in concentrations between 5 and $15 \%$ was proven to have a skin anti-aging effect by inducing the production of Col-1, and Col-3, as well as enzymes important for the production of collagen, and inhibitors of matrixmetalloproteinase (MMP) 1 (collagenase 1). Clinical studies have proven that the antioxidative protection is higher with the combination of vitamins $\mathrm{C}$ and $\mathrm{E}$ than with the vitamin $\mathrm{C}$ or $\mathrm{E}$ alone [17].

Niacinamide (vitamin $\mathrm{B}_{3}$ ) regulates cell metabolism and regeneration, and it is used in 5\% concentration as an anti-aging agent. Vitamin E ( $\alpha$-tocopherol) used as a component of skin products has antiinflammatory and antiproliferative effects in concentrations between 2 and 20\%. It acts by smoothing the skin and increasing the ability of the stratum corneum to maintain its humidity, to accelerate the epithelialization, and contribute to photoprotection of the skin. The effects are not as strong as with vitamins $\mathrm{C}$ and $\mathrm{B}_{3}$ [18].

The topical application of green tea polyphenols before UV exposure leads to an increase of the minimal erythema dose, decreases the number of Langerhans cells and reduces DNA damage in the skin [19]. Other botanicals that act as antioxidants are for example the isoflavones from soya.

Cell regulators, such as vitamin A derivatives, polypetides and botanicals, act directly on the collagen metabolism and stimulate the production of collagen and elastic fibers.

Vitamin A (retinol) and its derivates (retinaldehyde and tretinoin) are also a group of agents with antioxidant effects. They can induce the biosynthesis of collagen and reduce the expression of MMP 1 (collagenase 1). Retinol is, at the moment, the substance that is most often used as an anti-aging compound and, compared with tretinoin, causes less skin irritation [20]. Retinol has positive effects not only on extrinsic but also on intrinsic skin aging and has a strong positive effect on collagen metabolism. Tretinoin, a nonaromatic retinoid of the first generation, is approved for application as an anti-aging treatment in a concentration of $0.05 \%$ in the United States.

Invasive Procedures: There are various in-office procedures, most of which are intended to 'resurface' the epidermis: to remove the damaged epidermis and replace the tissue with remodelled skin layers and sometimes spur the formation of new collagen. It is possible that the potential of GF, cytokines and telomerase will eventually be harnessed via technological advancement and innovation in the burgeoning fields of tissue engineering and gene therapy in the nearest future [21].

Chemical Peels: Chemical peels are methods to cause a chemical ablation of defined skin layers to induce an even and tight skin as a result of the regeneration and repair mechanisms after the inflammation of the epidermis and dermis. Chemical peels are classified into three categories [22]. Superficial peels [ $\alpha-\beta-$, lipo-hydroxy acids 


\section{International Journal of Engineering Applied Sciences and Technology, 2019 Vol. 4, Issue 7, ISSN No. 2455-2143, Pages 255-263 \\ Published Online November 2019 in IJEAST (http://www.ijeast.com)}

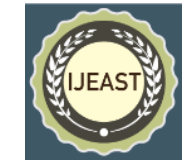

(HA), trichloroacetic acid (TCA) 10-30\%] exfoliate epidermal layers without going beyond the basal layer; medium-depth peels (TCA above 30 to $50 \%$ ) reach the upper reticular dermis; deep peels (TCA > 50\%, phenol) penetrate the lower reticular dermis. The depth of peeling depends not on the substance used only, but on its concentration, $\mathrm{pH}$ of the solution and time of application. A number of skin modifications have been reported after several weeks: epidermal architecture returns to normal, melanocytes are present and distributed uniformly, basal cells contain small melanin grains distributed homogeneously, the thickness of the basal membrane is homogeneous, in the dermis, a new sub epidermal band of collagen appears, elastic fibers form a new network, often parallel to those of collage [23]. If superficial peelings target the corneosomes, cause desquamation, increase epidermal activity of enzymes, lead to epidermolysis and exfoliation, medium-depth peels cause coagulation of membrane proteins, destroy living cells of the epidermis and, depending on the concentration, the dermis. Deep peels coagulate proteins and produce complete epidermolysis, restructure of the basal layer and restoration of the dermal architecture The depth of peel correlates with the potential side-effects, like hyperpigmentation, solar lentigines, risk of postoperative infections, especially herpetic ones. The mechanism by which the chemical peel takes effect is not clearly elucidated [24]. An increase in collagen fiber content, water and GAG in the dermis has been reported. There is a suggestion that improvements in skin elasticity and wrinkles after chemical peeling can be attributed to increase of Col-1 with or without Col-3, elastic fibers, as well of a dense rearrangement of collagen fibers [25].

Visible Light Devices (IPL, Lasers, RF for the Skin Rejuvenation, Resurfacing and Tightening): Nonablative skin rejuvenation or "subsurfacing" comes as a low risk and short downtime technology which can improve aging structural changes without disruption of cutaneous integrity. The mechanism of action is supposed to be a selective, heat induced denaturalization of dermal collagen that leads to subsequent reactive synthesis. Nonablative skin rejuvenation is not a precise term since rejuvenation is a controlled form of skin wounding aimed at achieving a more youthful appearance after the wound heals [26].

Treatment of photoaged skin has been divided into treatment of ectatic vessels and erythema, irregular pigmentation, and pilosebaceous changes (Type I) and into the improvement of the dermal and subcutaneous senescence (Type II). The epidermis and superficial dermis can be selectively damaged by two basic mechanisms: (a) by targeting discrete chromophores in the dermis or at the dermalepidermal junction or (b) by utilizing mid infrared (IR) lasers [27].

The devices for treatment of vascular and/or pigment irregularities include lasers emitting light at 532-, 585-, 595-, 755-, 800-, and 1064-nm wavelengths as well as filtered light generated by IPL systems equipped with different cut-off filters (Fig. 1). Lasers emitting 1,320, 1,450, and 1,540 $\mathrm{nm}$ using interstitial and intracellular water as target chromophores and pulsed dye lasers (PDL) using oxyhemoglobin as the primary chromophore are now employed for Type II photo rejuvenation only. The clinical efficacy of these nonablative modalities are weaker than that of the ablative methods, however, new collagen formation and clinically observable improvement in wrinkles can be observed. Reduction of facial wrinkles by using IPL devices has shown less effect comparing to laser technology, but for type I photo rejuvenation, IPL systems have in general shown considerably better results than laser systems operating at subpurpuric energy levels [28].

Ultrastructural and histological analysis confirmed effectiveness of absorption of light $(532,585,595$, with or without 1064-nm Nd:YAG laser) in the blood vessels of the superficial dermis, resulting in the release of inflammatory mediators and GF into the interstitium followed by stimulated fibroblast activity and initiation of tissue repair and enhanced collagen and elastin neoformation replacing the originally damaged elastic tissue. An increase in grenz zone thickness, monoclonal chondroitin sulfate and III procollagen staining as well as quantification of Col-1 was measured after couple of treatments with PDL. The increase in dermal collagen has also been confirmed by noninvasiveultrasonographic analysis and radioimmunoassay [30]. Nonablative skin rejuvenation should not yet be considered an alternative for laser resurfacing. However there are interesting data showing comparative histological changes between the ablative and nonablative modalities [29].

Histological sections of skin before and after treatment with the different IPL devices have shown the formation of new collagen in the papillary and reticular dermis, as well as an increase in the number of fibroblasts and proportional decrease in the amount of solar elastosis is also usually found [31]. If vascular and/or pigment disturbances improvement are immediate, the collagen remodeling response is delayed and maximum results are seen only between 3 and 12 mo after treatment [26].

Laser resurfacing has been shown to be effective in counteracting photoaging through entire epidermal ablation, collagen shrinkage, stimulation of 


\section{International Journal of Engineering Applied Sciences and Technology, 2019 Vol. 4, Issue 7, ISSN No. 2455-2143, Pages 255-263 \\ Published Online November 2019 in IJEAST (http://www.ijeast.com)}

neocollagenesis, extensive dermal remodeling, regeneration of cellular organelles and intercellular attachmentsbutparallelly, results in long recovery time are associated with risks of severe long lasting side effects, such as persistent erythema, hypo- or hyperpigmentation, infection or scarring [32].

Although the underlying molecular changes induced by different ablative and non-ablative as well as thermal and non-thermal skin rejuvenation treatments are not fully understood, there are investigations suggesting important roles of heat shock proteins (HSP), transforming growth factor $\beta$ (TGF- $\beta$ ), different MMPs, synthethases, hyals and hyaluronic acid (HA). Type I and type III procollagen mRNA was also elevated for at least 6 mo [33].
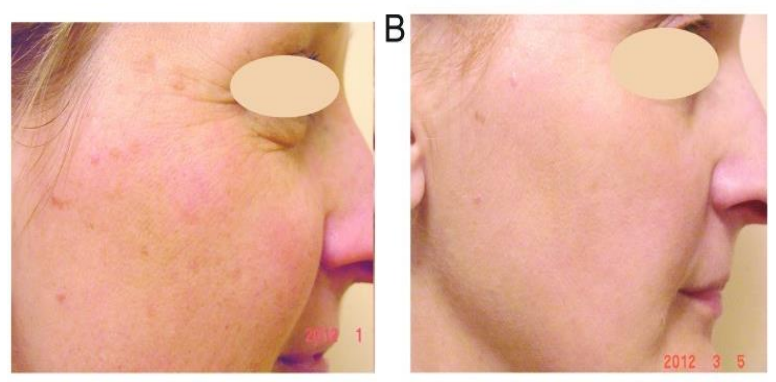

Fig. 1. 45-y-old female with signs of photoaged skin: dyschromia of the skin, multiple lentigines. (A) before, (B) after one treatment with IPL with $550 \mathrm{~nm}$ cut-off filter.

Injectable Skin Rejuvenation and Dermal Fillers: The goal of skin biorejuvenation is to increase the biosynthetic capacity of fibroblasts, inducing the reconstruction of an optimal physiologic environment, the enhancement of cell activity, hydration, and the synthesis of collagen, elastin and HA (hyalorunic acid). The desired effect could be achieved by the microinjections in the superficial dermis of products containing only one active ingredient or cocktails of different compounds which are perfectly biocompatible and totally absorbable: HA, vitamins, minerals, nutrients, hormones, GF, amino acids, autologous cultured fibroblasts, homeopathic products, etc. The distinct formulations can induce strikingly divergent molecular and cellular processes in fibroblasts in vitro [34]. (Fig. 1)

HA from bacterial fermentation), synthetic or pseudo-synthetic implants (silicone, polymethacrylate microspheres, poly-L-lactic acid, calcium hydroxylapatite microspheres suspended in aqueous polysaccharide gel, alkyl-imide gel polymer). These may be grouped into temporary, semipermanent (lasting between 1-2 y), or permanent materials (lasting longer than $2 \mathrm{y}$ ).

GAG and particularly HA or hyaluronan are major components of the cutaneous extracellular matrix involved in tissue repair of all animal tissues.HA exhibits no species or tissue specificity. As a physical background material, it has functions in space filling, lubrication, shock absorption, and protein exclusion. In addition, $\mathrm{HA}$ has been implicated as a regulator of cell proliferation and locomotion. Injection of HA is thought to promote skin rejuvenation by increasing both hydration and fibroblast activation. HA injected into the skin can stimulate fibroblasts to express Col-1, MMP-1 and tissue inhibitor of matrix metalloproteinase-1 (TIMP-1) as well as is participating in wound healing, modulation of inflammatory cells, interaction with proteoglycans of the extracellular matrix, and scavenging of FR [35]. All these features of HA have made it to be useful as an ideal structural compound and have raised injections of HA products to the most acceptable and scientifically investigated "gold standard" procedures for skin rejuvenation and augmentation (Fig. 2).

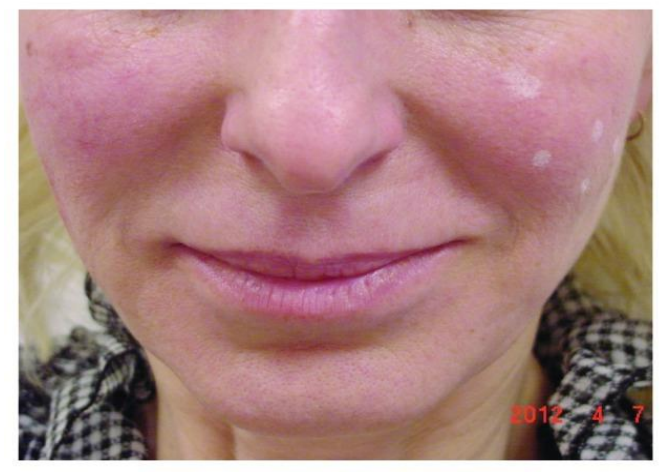

Figure 2. Patient showing the difference of the nasolabial fold: non-treated left side (with site marks for planned HA injection) and right side straight after injection of only $0.5 \mathrm{ml}$ of nonanimal stabilized cross-linked HA

Natural HA has a half-life in tissue of only 1 to $2 \mathrm{~d}$ before undergoing aqueous dilution and enzyme degradation in the liver to carbon dioxide and water. Produced from bacterial (Staphylococcus equine)fermentation and modified by chemical cross-linking to improve their resistance to enzymatic degradation and prolong their effect, non-animal reticulated HA fillers are more pure, more viscous, usually well tolerated and rarely elicit adverse and immunological reactions. The duration of effect for HA fillers ranges from 3 to 12 mo. The long-lasting dermal fillers maintain the position 1-2 y or even more [36].

Modern HA fillers differ in the particulate size, cross-linking and the type of cross-linking agent used in the HA; phasic structure-mono/biphasic, concentration of HA and presence of an anesthetic agent in each syringe. One of long-lasting synthetic semi-permanent dermal fillers is calcium hydroxyl 


\section{International Journal of Engineering Applied Sciences and Technology, 2019 Vol. 4, Issue 7, ISSN No. 2455-2143, Pages 255-263 \\ Published Online November 2019 in IJEAST (http://www.ijeast.com)}

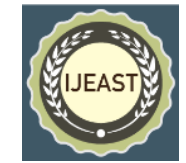

apatite based filler (CaHA) suspended in an aqueous carboxymethylcelluose gel carrier. The CaHA particles act as a scaffold for new tissue formation and stimulate collagen formation around the microspheres leading to a thickening of the dermis over time. The spherical CaHA particles are gradually phagocytosed, degraded as calcium and phosphate and eliminated via the renal system. CaHA is biocompatible with an identical composition to bones with a low potential for antigenicity, foreign body reaction, and minimal inflammatory response. No osteoblast activity has been observed in soft tissue [37].

Autologous Platelet-Rich Plasma (PRP): Autologous Platelet-rich Plasma (PRP) has attracted attention for skin rejuvenation. PRP is derived from fresh whole blood, which contains a high concentration of platelets. Various GF, including platelet-derived growth factor (PDGF), transforming growth factor (TGF), vascular endothelial growth factor (VEGF), and insulin-like growth factor (IGF), are secreted from the $\alpha$ granules of concentrated platelets activated by aggregation inducers. These factors are known to regulate processes including cell migration, attachment, proliferation and differentiation, and promote extracellular matrix (ECM) accumulation by binding to specific cell surface receptors. It has been shown that PRP may induce the synthesis of collagen and other matrix components by stimulating the activation of fibroblasts, thus, rejuvenating the skin. However, the molecular mechanisms underlying PRP-inducing wound healing processes are still largely unknown and experimental studies confirming the effects of PRP on aged fibroblasts are very limited [38].

Botulinum Toxin: Botulinum toxin (BTX) has no effect on skin texture and cannot discontinue the skin aging process. However, regular BTX injections can slow down the visible aging process by helping in the management of certain dynamic facial lines and wrinkles (Fig. 3). Current treatment options of exaggerated frown lines, glabellar lines or crow feet such as surgery or implants, do not address the underlying cause of these lines, namely the excessive nerve stimulation. The mechanism of action of BTX makes it an ideal agent to target the major cause of these dynamic lines [39]. (Fig.3)
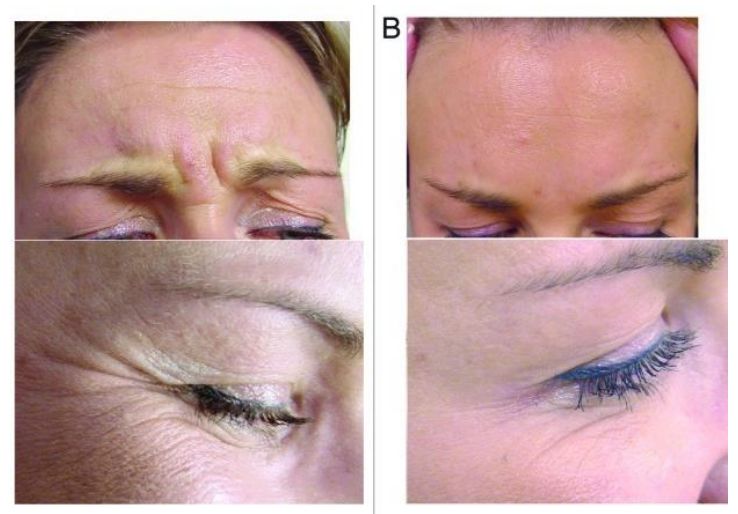

Fig. 3. Patient showing glabellar and crow's feet wrinkles. (A) pre-injection, (B) after injection with botulinum toxin.

The toxin binds to presynaptic neurons of selected muscles rapidly (under an hour) and specifically. Clinically reversible chemical denervation and selective muscle relaxation or paralysis starts after 24 to $48 \mathrm{~h}$ and may not be completed for up to 2 weeks. In muscle, approximately on day 28 , nerve sprouts mediate a partial restoration and new neuromuscular junctions are formed in the vicinity of the old junctions. Another factor explaining the regaining of muscle function could be an increase in the area of muscle membrane sensitive to acetylcholine. On days 62-91, complete muscle function recovery can be demonstrated.

Muscular changes in the form of atrophy were demonstrated in animal studies, and were completely reversible after 4-6 mo. In human muscle, no lasting atrophy could be detected even after repeated injections, only a predominance of type I fibers. The usual duration of effect is 3-6 mo with individual variations.

Dosing of BTX-A is essential in achieving precise and predictable effects. The biological activity given in mouse units (MU) and the weight of the molecule is not associated with the dosage. One MU is equivalent to the amount of toxin at which, after intraperitoneal administration, half of the poisoned Swiss-Webster mice die (50\% lethal dose; LD50). The amounts of BTX-A used for the treatment are 25-100 times less than the LD50, so that the FDA classifies BTX-A as therapeutically safe. BTX-A does not cross the blood-brain barrier or pass through the skin [40]

The incidence of complications in many cases depends on the proper application and the qualification of the physician. However, it has always to be considered that the benefits of this treatment are transient and repeated injections are necessary for a long-term effect.

Hormone Replacement Therapy (HRT): It is well known that there is a progressive decrease of hormone synthesis with age. Levels of growth hormone $(\mathrm{GH})$ and insulin-like growth factor-1 (IGF-1), melatonin (nocturnal), TSH, thyroid hormones (T3), dehydroepiandrosterone (DHEA) (sulphated form and its urinary 17-ketometabolites), estrogens and testosterone are progressively decreasing. The main hormonal deficits in humans are menopause, andropause and partial androgen deficiency of the aging male. DHEA substitution has been proven to lead 


\section{International Journal of Engineering Applied Sciences and Technology, 2019 \\ Vol. 4, Issue 7, ISSN No. 2455-2143, Pages 255-263 \\ Published Online November 2019 in IJEAST (http://www.ijeast.com)}

to an improvement of body condition, sexual activity, bone density, and well-being [41].

Melatonin has been shown to have a favourable influence on the aging process, because it has an inverse effect with regard to body weight; food restriction raises the levels of melatonin and decreases its age-related decrease. With increasing age comes a decrease of melatonin production, which may have a connection to sleep disorders suffered by elderly people. It also has been shown that melatonin can prevent tumour development and growth. Interestingly, a study showed that patients with tumours had decreased levels of melatonin compared with healthy individuals [42].

HRT with estrogen and progesterone has been long considered to have anti-aging effects; results of larger studies though, particularly of the Women's Health Initiative, have shown that an anti-aging effect is not necessarily to be expected. On the contrary, HRT has been accused to have a higher cardiovascular risk and increase of the risk of breast cancer. However, it has clear, positive preventive effects on osteoporosis, and an early, low-dose estrogenmonotherapy can be considered to have advantages [43].

\section{CONCLUSIONS}

While natural aging is genetically determined, extrinsic aging can be prevented. Aesthetic dermatology should contribute to "healthy aging" not only in cosmetic means by trying to erase time vestiges in skin but by also playing a significant part in prevention, regeneration, and delaying of skin aging combining knowledge of possible local and systemic therapy, instrumental devices and invasive procedures, filling the lack of scientific investigations and becoming one of the important focuses of the aging research.

\section{REFERENCES}

1. Cevenini E., Invidia L, Lescai F, Salvioli S, Tieri P, Castellani G. (2008). Human models of aging and longevity. Expert Opin BiolTher, 1393-405.

2. Uitto J. (1997). Understanding premature skin aging. N Engl J Med. 337:1463-5.

3. Yaar M, Gilchrest BA. (1999). Aging of skin. In Fitzpatrick's Dermatology in General Medicine Vol 2, 5th edn. McGraw-Hill:New York, 1697-1706.

4. Audonneau JL, Jeanmaire C, Pauly G. (1999). A histological study of human wrinkle structures: comparison between sun-exposed areas of the face, with or without wrinkles, and sun-protected areas. Br J Dermatol. 140:1038-47.

5. Shuster S, Black MM, McVitie E. (1975). The influence of age and sex on skin thickness, skin collagen and density. $\mathrm{Br} \mathrm{J}$ Dermatol. 93:639-43.

6. Elsner P, Maibach HI. (2005). Cosmeceuticals and Active Cosmetics: Drugs versus Cosmetics (2nd edn). Marcel Dekker: New York.

7. Baumann L. (2007). Skin ageing and its treatment. J Pathol. 211:241-51.

8. Calasanti TM, Slevin KF, King N. (2006). Ageism and feminism: from 'et cetera' to center. NWSA J.18:13-30.

9. Bayer K. (2005). Cosmetic surgery and cosmetics: redefining the appearance of age generations. 13-8.

10. Holstein MB, Minkler M. (2003). Self, society, and the new gerontology. Gerontologist. 43:787-96.

11. Dierickx CC, Anderson RR. (2005). Visible light treatment of photoaging. Dermatol Ther. 18:191-208.

12. Tabata N, O'Goshi K, Zhen YX, Kligman AM, Tagami H. (2000). Biophysical assessment of persistent effects of moisturizers after their daily applications: evaluation

of cosmotherapy. Dermatology. 200:308-13.

13. Lübbe J. (2000). Evidence-based corneotherapy. Dermatology. 200:285-9.

14. Trautinger F. (2001). Mechanisms of photodamage of the skin and its functional consequences for skin ageing.ClinExpDermatol. 26:573-7.

15. Marini A.(2011). Beauty from the inside. Does it really work? Hautarzt. 62:614-7.

16. Bissett DL, Miyamoto K, Sun P, Li J, Berge CA. (2004). Topical niacinamide reduces yellowing, wrinkling, red blotchiness, and hyperpigmented spots in aging facial skin. Int $\mathrm{J}$ Cosmet Sci. 26:231-8.

17. Kerscher M, Buntrock H. (2011). Antiaging creams. What really helps? Hautarzt. 62:607-13.

18. Zhai H, Behnam S, Villarama CD, ArensCorell M, Choi MJ, Maibach HI. (2005). Evaluation of the antioxidant capacity and preventive effects of a topical emulsion 
and its vehicle control on the skin response to UV exposure. Skin Pharmacol Physiol. 18:288-93.

19. Elmets CA, Singh D, Tubesing K, Matsui M, Katiyar S, Mukhtar H. (2001). Cutaneous photoprotection from ultraviolet injury by green tea polyphenols. J Am AcadDermatol. 44:425-32.

20. Kafi R, Kwak HS, Schumacher WE, Cho S, Hanft VN, Hamilton TA. (2007). Improvement of naturally aged skin with vitamin A (retinol) Arch Dermatol. 143:606-12.

21. Ostler EL, Wallis CV, Aboalchamat B, Faragher RG. (2000). Telomerase and the cellular lifespan: implications of the aging process. J PediatrEndocrinolMetab. 13(Suppl 6):1467-76.

22. Monheit GD, Chastain MA. (2001). Chemical peels. Facial Plast Surg Clin North Am. 9:239-55.

23. Brown AM, Kaplan LM, Brown ME. (1960). Phenol-induced histological skin changes: hazards, technique, and uses. $\mathrm{Br}$ J Plast Surg. 13:158-69.

24. Han SH, Kim HJ, Kim SY, Kim YC, Choi GS, Shin JH. (2011).Effects of chemical peeling in photoaged hairless mice. Int $\mathbf{J}$ Dermatol. 50:1075-82.

25. Butler PE, Gonzalez S, Randolph MA, Kim J, Kollias N, Yaremchuk MJ. (2001). Quantitative and qualitative effects of chemical peeling on photo-aged skin: an experimental study. PlastReconstr Surg. 107:222-8.

26. Dierickx CC, Anderson RR. (2005). Visible light treatment of photoaging. DermatolTher. 18:191-208.

27. Hardaway CA, Ross EV. (2002). Nonablative laser skin remodeling. DermatolClin. 20:97-111.

28. Tanghetti E, Sherr E. (2003).Treatment of telangiectasia using the multi-pass technique with the extended pulse width, pulsed dye laser (Cynosure V-Star) J Cosmet Laser Ther. 5:71-5.

29. Alam M, Dover JS, Arndt KA. (2003). Treatment of facial telangiectasia with variable-pulse high-fluence pulsed-dye laser: comparison of efficacy with fluences immediately above and below the purpurathreshold. Dermatol Surg. 29:6814, discussion 685.

30. Bjerring P, Clement M, Heickendorff L, Lybecker H, Kiernan M. (2004). Dermal collagen production following irradiation by dye laser and broadband light source. $\mathbf{J}$ Cosmet Laser Ther. 4:39-43.

31. Dahiya R, Lam SM, Williams EF. (2003). A systematic histological analysis of nonablative laser therapy in a porcine model using the pulsed dye laser. Arch Facial Plast Surg. 5:218-23.

32. Lowe NJ, Lask G, Griffin ME, Maxwell A, Lowe P, Quilada F. (1995). Skin resurfacing with the Ultrapulse carbon dioxide laser. Observations on 100 patients. Dermatol Surg. 21:1025-9.

33. Hantash BM, Bedi VP, Kapadia B, Rahman Z, Jiang K, Tanner H. (2007). In vivo histological evaluation of a novel ablative fractional resurfacing device. Lasers Surg Med. 39:96-107.

34. Jager C, Brenner C, Habicht J, Wallic R. (2011). Bioactive reagents used in mesotherapy for skin rejuvenation in vivo induce diverse physiological processes in human skin fibroblasts in vitro - a pilot study. ExpDermatol. 21:70-80.

35. Jiang D, Liang J, Noble PW. (2007). Hyaluronan in tissue injury and repair. Annu Rev Cell Dev Biol. 23:43561.

36. Eppley BL, Dadvand B. (2006). Injectable soft-tissue fillers: clinical overview. Plast Reconstr Surg. 118:98e-106e.

37. Tzikas TL. (2008). A 52-month summary of results using calcium hydroxylapatite for facial soft tissue augmentation. Dermatol Surg.34(Suppl 1):S9-15.

38. Freymiller EG. (2004). Platelet-rich plasma: evidence to support its use. J Oral Maxillofac Surg. 62:1046-1047.

39. Böni R, Kreyden OP, Burg G. (2000). Revival of the use of botulinum toxin: application in dermatology.Dermatology. 200:287-91.

40. Becker-Wegerich P, Rauch L, Ruzicka T. (2001). Botulinum toxin A in the therapy 
International Journal of Engineering Applied Sciences and Technology, 2019

Vol. 4, Issue 7, ISSN No. 2455-2143, Pages 255-263

Published Online November 2019 in IJEAST (http://www.ijeast.com)

of mimic facial lines. ClinExpDermatol. 26:619-30.

41. Morales AJ, Nolan JJ, Nelson JC, Yen SS. (1994). Effects of replacement dose of dehydroepiandrosterone in men and women of advancing age. J ClinEndocrinolMetab. 78:1360-7.

42. Wetterberg L, Bergiannaki JD, Paparrigopoulos T, von Knorring L, Eberhard G, Bratlid T. (1999). Normative melatonin excretion: a multinational study. Psychoneuroendocrinology. 24:209-26.

43. Heutling D, Lehnert H. (2005). [Hormone therapy and menopause] Dtsch Med Wochenschr.130:829-34. 